\title{
Fine Structure of Nasal and Oral Cavities in Dog and their Sensory Innervation, especially on the Intraepithelial Fibres
}

\author{
By \\ Yoshihide Abe \\ (阿 部 芳 秀) \\ From the Anatomical Laboratory of Prof. H. Seto, \\ Tohoku University, Sendai
}

(Received for publication, September 12, 1953)

The fact that, in the alimentary canal in man, its entrance, the oral cavity, and its exit, the anus, and in the respiratory tract, its entrance, the vestibulum nasi, are rich in nerves, especially in sensory nerves, has been clearly demonstrated by many studies in this laboratory. 'The terminations of these sensory fibres are chiefly formed in the tunica propria of the mucous membrane, especially in the papillae, but in places, the sensory fibres penetrate further in to the epithelium, to end there as intraepithelial fibres.

There have been reported many studies on these sensory terminations in the past, but owing to the extreme difficulty of adequately staining nerve fibres, there seems to exist many points of dubious validity in these studies, in particular, on the intraepithelial fibres. For example, intraepithelial fibres have been found in parts where no such fibres had to exist, such as the epidermis of haired skin or the outer follicle sheath, and even in the parts where intraepithelial fibres might be expected, the delineated intraepithelial fibres represented nothing but illusory artefacts. Such unreliable informations were rather regrettably frequent in the past literature.

Since about 20 years ago Prof. Seto and his many co- workers in this laboratory have studied on the sensory terminations in the above mentioned propria as well as the epithelium, with human mucosa as objective, so that the research on this subject seems to have reached a stage of final completion.

These studies have elucidated, among other things, that intraepithelial fibres are never formed in the incessantly desquamating cornified stratified flat epithelium, such as the epidermis of haired skin, and that they are found only in limited areas of the non-cornified stratified flat epithelium, for instance in vestibulum nasi, transitional part of lips, - gums, hard palate and anus. They are also found in the ciliated epithelium, but again in limited areas, namely, in larynx, pharynx, trachea and 
bronchi, but not in cavum nasi. In the stratified columnar epithelium they are found in urethra, and in the transitional epithelium, for example in vesica urinaria and urethra. Besides, in the taste buds and in the common epithelium between glans penis or glans clitoridis and the inner plate of praeputium in embryonic time, conspicuous intraepithelial fibres are observable.

The nerve fibres penetrating into the epithelium are never vegetative, but always sensory in nature, so that it may be readily surmised that the areas rich in such fibres are extremely sensitive. The character and the formation of the intracpithelial fibres are more or less different according to the areas of their existence.

As described above, the knowledge on the sensory terminations in the mucous membrane has been greatly amplified by such studies in this laboratory. The author of this report has also been granted the opportunity of succeeding them in their study, with the sensory terminations, especially the intraepithelial fibres, in the mucosa of the nasal cavity with the snout and the oral cavity in dog, one month of age, as my objective. My materials were fixed for a long time in 10\% neutral formol, cut into $30-40 \mu$ frontal and sagittal frozen sections, and stained with Seto's silver impregnation. The series of the very beautifully stained preparations thus obtained I subjected to a minute examination and arrived at the observations reported in the following.

\section{Individual Observations}

Innervation of mammalian nasal cavity:

I could not find any literature on this subject, while on the innervation of the human vestibulum nasi and pars respiratoria of cavum nasi, Seto and Sada ${ }^{1)}$ (1944) and Sato2) (1949) have respectively published detailed and dependable studies. According to Sato, ${ }^{2}$ in the subepithelial connective tissue of pars respiratoria of human nasal cavity there are found numerous vegetative nerve fibres, but only a few of sensory fibres originating from the trigeminal nerve. The terminations of these latter fibres are of the simplest formation, and are located mostly in the subepithelial propria, almost none of them penetrating into the epithelium itself.

In the mucosa of the human vestibulum nasi (Seto and Sada)1), however, we can find beside branched terminations also corpusclar terminations in the propria, especially in its papillae and further intraepithelial fibres in many places. Since the distribution of sensory fibres generally stands in close relation with the minute structure of the mucous membrane, Seto and Sada also here in vestibulum nasi conducted a minute research on its fine structure, especially on the epithelium and the papilla formation, and in accordance with many studies in this laboratory, proved that the more remarkable the development of the papillae, the better is the sensory supply here too. 
The human vestibulum nasi is divisible into pars cutanea with vibrissae and pars mucosa, and the latter again into the corneous and the non-corneous parts. Besides, a narrow transitional zone covered with stratified columnar epithelium lies between the vestibulum and pars respiratoria of cavum nasi. In the corneous mucosa part, the epithelium is much thicker than that in pars cutanea, and is composed of a stratified flat epithelium provided with corneous plate instead of stratum corneum. Samall papillae formed by the subepithelial connective tissue are arranged regularly against the epithelium. The noncorneous mucosa part is covered by a stratified flat epithelium without corneous plate, and the papilla formation is here so very poor, that the basal surface of the epithelium becomes almost smooth and even upon approaching the transitional zone.

The development of the sensory fibres is especially marked in the corneous mucosa part where the papillae are also in advanced formation, and corpusclar terminations and intraepithelial fibres of considerable size are found existing. In the non-corneous mucosa part, sensory fibres are much poorer in development, but the formation of minute branched terminations consisting of a few rami is often found in the epithelium.

Now, the results of my studies upon the minute structure of the mucous membrane of the canine nasal cavity are in good accordance with those in man. The mucous membrane of pars respiratoria of cavum nasi in dog is lined with stratified ciliated epithelium, nasal glands and well-developed venous plexus are found in the tunica propria and submucosa which are almost indivisible from each other, but the smooth muscle fibres found in human cavity are not found here. In this respiratory part there are found rather thick nerve bundles running through the submucosa, mostly composed of minute nonmedullated vegetative fibres, which gradually branch out and anastomose mutually, finally to pass into the terminalreticulum and to distribute around the blood vessels. On the contrary, the sensory fibres found in the nerve bundles are much smaller in number. They run into the tunica propria directly beneath the epithelium and end there as unbranched or simple branched terminations. No fibres have ever been found to penetrate further into the epithelium.

The fine structure of vestibulum nasi in $\operatorname{dog}$ is much the same with that in man, but some interesting differences are found in that the canine vestibulum nasi lacks pars cutanea with vibrissae, and its front part passes partly into the suout mentioned below, partly into the haired skin. The distribution area of the corneous and the non-corneous mucosa parts is very varied according to the position, but generally speaking, the vestibulum seems to be almost divided by both the mucosa parts into the anterior and the posterior halves.

The epithelium in the corneous mucosa part is much thicker than the epidermis of the haired skin, consisting of stratified flat epithelium covered by corneous plate. The propria forms low, small papillae arranged regularly against the basis of the epithelium. The non-corneous mucosa part is covered by a stratified flat epithelium without corneous plate, the basis of which grows gradually smoother as the deeper part is approached. It must be specially 
mentioned that this vestibulum mucosa has much poorer developed nasal glands than in man, which are distributed very sporadically, and weakly developed venous plexus.

Also in canine cavum nasi, much more nerve bundles are found penetrating the submucosa of the vestibulum nasi than that of the respiratory part. These bundles contain a large number of thick medullated sensory fibres beside the thin vegetative fibres. The bundles gradually branch out and the vegetative fibres therein finally pass into the terminalreticulum, chiefly spreading out in the tunica propria, while the sensory fibres run into the corneous, rather than into the non-corneous, mucosa part in a far greater number, to end mostly in branched terminations in the subepithelial connective tissue, especially in the papillae. Such corpusclar terminations, as found by Seto and $\mathrm{Sada}^{1}$ in human vestibulum were not to be seen in my baby dogs. But when we consider that the corpusclar endings, excepting the Pacinian bodies, are yet unformed in human embryos, it may be supposed that such bodies are formed later on also in dog, gradually as it grows to maturity.

Seto and $\mathrm{Sada}^{11}$ have found a comparatively large number of intraepithelial fibres in the epithelium of human vestibulum, and classified them into branched terminations composed of minute fibres and short unbranched terminations consisting of thick fibres coming in from the top of papillae and reaching the upper layer of the epithelium. Also in my dog vestibulum, I found intraepithelial fibres even in a larger number than in man, especially numerous at the entrance of the vestibulum. In outer skin covered by desquamating corneous epithelium, no intraepithelial fibre is ever found, but it is of interest that such fibres appear as soon as we pass from the haired skin into the vestibulum.

Some of these intraepithelial fibres originate in the branched terminations formed in the papillae (Figs. 1 and 2), and others are represented by sensory fibres running singly through the papillae and penetrating directly into the epithelium. Some of the latter end in unbranched terminations without branching out (Fig. 3), while others end in branched terminations after ramifying in the epithelium (Fig. 4). These fibres are in most cases smooth-surface, thin and uniform in size throughout, but some are thicker, as shown in Fig. 2. They run through or between epithelial cells and end sharply or more rarely in small nodes (Fig. 3) in any of its layers. No such short but very stout unbranched intraepithelial fibres running out of the top of papillae, as found by Seto and Sada') in human vestibulum nasi, was ever found in my baby dogs.

Innervation of the snout: The snout, peculiar to the mammals, is very rich in sensory nerve fibres, and having particularly well-developed intraepithelial fibres, has offered an appropriate theme for many investi- 
Fig. 1

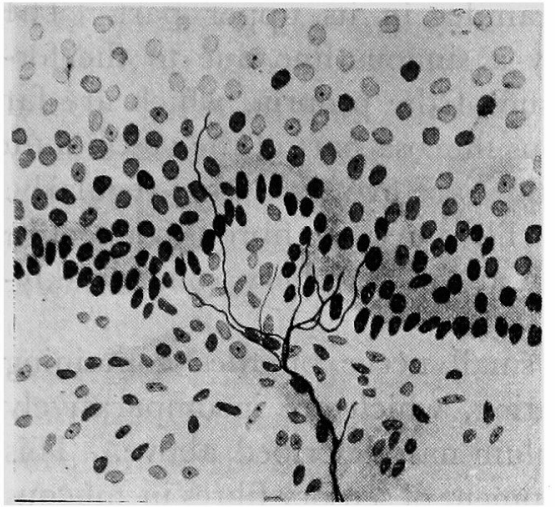

Fig. 2

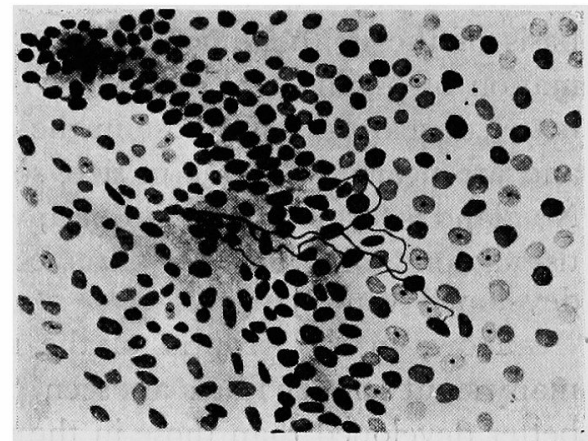

Fig. 1. Branched sensory termination formed in a papilla in the corneous mucosa part near the entrance of the vestibulum nasi in dog. Seto's impregnation, $\mathrm{X} 600$, reduced to $\frac{1}{2}$.

Fig. 2. Ditto. The terminal fibres are of comparatively thick nature. Same staining, $\mathrm{X} 600$, reduced to $\frac{1}{2}$.

Fig. 3

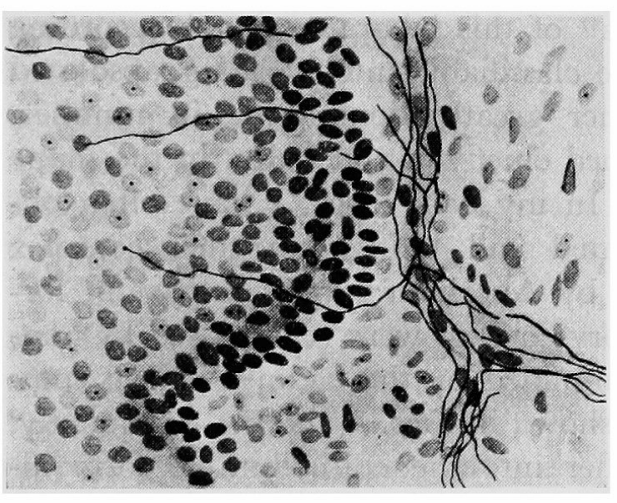

Fig. 4

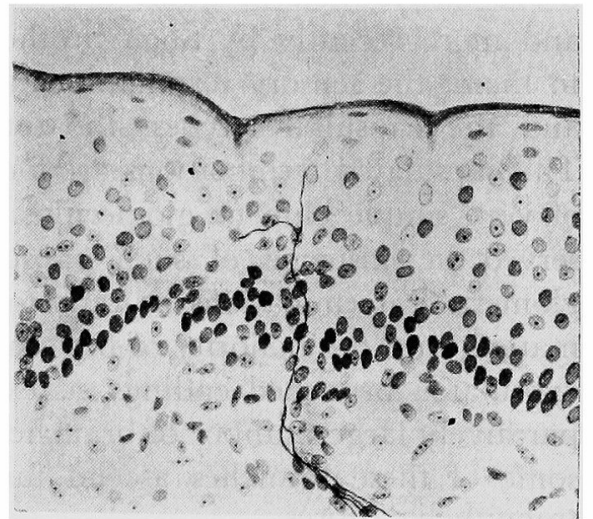

Fig. 3. Unbranched intraepithelial fibres ending sharply or in node originated in singly running sensory fibres seen in the corneous mucosa part of vestibulum nasi in dog. Same staining, X 400, reduced to $\frac{1}{2}$.

Fig. 4. Bifurcated intraepithelial fibre found in the corneous mucosa part of vestibulum nasi in dog. Same staining, X 400, reduced to $\frac{1}{2}$.

gators in the past. I will preface my description on the innervation of this part with a histological picture thereof, because it has a close bearing on the nerve distribution. The snout is presumed to represent the foremost part of vestibulum nasi from the ontogenetic point of view. In fact, the epithelium thereof is very similar to that of the corneous mucosa part of the vestibulum, consisting of stratified flat epithelium having corneous 
plate, somewhat larger in height than that in the vestibulum and containing a large quantity of pigment granules in its upper part. The basis of the epithelium is represented by a sinuous line due to the formation of papillae by the connective tissue of the propria, which are far better developed than those in the corneous mucosa part of the vestibulum and are nearly the same in size, so that they are arranged very regularly. No gland is formed in the propria, which is filled up with fibrous connective tissue, containing in some parts, especially at the periphery, some weaklydeveloped venous plexus.

Now, in the propria of the snout, small nerve bundles containing many stout sensory fibres are seen penetrating, which are incomparatively better developed than those in the vestibulum nasi described above. This fact again proves the thesis that the development of sensory fibres in mucous membrane covered by stratified flat epithelium is in proportion to the development of papillae, as has been established by many researches completed in this laboratory.

These sensory fibres run into the tunica propria, in particular, into the papillae, to end there chiefly in branched terminations. The sensory nerve distribution in adult $\operatorname{dog}$ has been studied by Ábrahám ${ }^{3)}$ (1931) and more recently by Suga brothers ${ }^{4}$ of this laboratory, and according to them, the sensory terminations are classifiable into the non-capsulated and the capsulated types, the former greatly dominating in number. The capsulated terminations are formed chiefly of end bulbs, in the shape of very simple club or glomerulus. In my baby dogs, I have often observed the existence of such capsulated end bulbs, but such complex glomerular terminations as reported by Ábrahám ${ }^{3)}$ were not yet in formation, and the majority of the sensory endings were represented by noncapsulated branched endings, as exemplified by the endings with a comparatively large number of branches shown in Figs. 5 and 6 . Frequently, some of these branches ascend further into the epithelium to form intraepithelial fibres.

The intraepithelial fibres in mammalian snouts have been the objectives of many past studies by Szymonowicz, ${ }^{5}{ }^{5}$ Trejakoff, ${ }^{6)}$ Merkel, ${ }^{7}$ Botezat $^{81}$ Kadanoff, ${ }^{91}$ Boeke, ${ }^{10)}$ Akkeringa, ${ }^{11}$ ) Hashizume, ${ }^{12}$ Huss, ${ }^{13)}$ Cybulsky, ${ }^{14)}$ Momono and Tsuda, ${ }^{15}$ but the majority of these studies were made on pigs, succeeded by studies on moles, cows and rabbits, only few, such as by Ábrahám ${ }^{31}$ in older times and by Suga brothers ${ }^{11}$ recently, being conducted with dogs as objectives. Botezat ${ }^{8}$ was successful in pointing out the existence of intraepithelial fibres in his methyleneblue stained preparations, but owing to the imperfection of his staining method, has missed to bring out the true nature of intraepithelial fibres. The results obtained by Ábrahám ${ }^{3)}$ by use of silver method have been 
more commendable, but the sensory fibres are much too thick in his figures. Suga brothers ${ }^{4)}$ failed to find intraepithelial fibres. This is perhaps due to their examining only the upper part of the snout rich in pigment granules.

According to my studies, numerous intraepithelial fibres were found to exist in the epithelium of the canine snout, which are oftener branched than unbranched at that. These intraepithelial fibres include those represented by the rami of the branched terminations formed in the papillae penetirating further into the epithelium (Figs. 5 and 6 ), and those originating in single fibres running into the epithelium and branching out there (Figs. 7, 8 and 9), as was the case in the vestibulum nasi.

These intraepithelial fibres are either thin (Fig. 8) or comparatively thick in nature (Figs. $5,6$ and 7$)$, as in the mucosa part of the vestibulum, but I could not find any such stout fibre as reported by Abrahám. ${ }^{31}$ The fibres run through or between the epithelial cells and end sharply, or sometimes in small nodes, in the upper layer of the epithelium. However, it is not at all rare that they terminate in the basal or the middle layer of the epithelium.

Of the innervation of the mucous membrane of the canine oral cavity, $\mathrm{Okano}^{16)}$ of this laboratory has previously reported in detail on his study on its lingual mucosa, so I will report only on the other parts, that is, on the lips, the cheeks, the gums and the palate mucosa. The studies hitherto reported on the innervation of the dog oral cavity have been centered on the tongue and the hard palate, but only few of them touching 

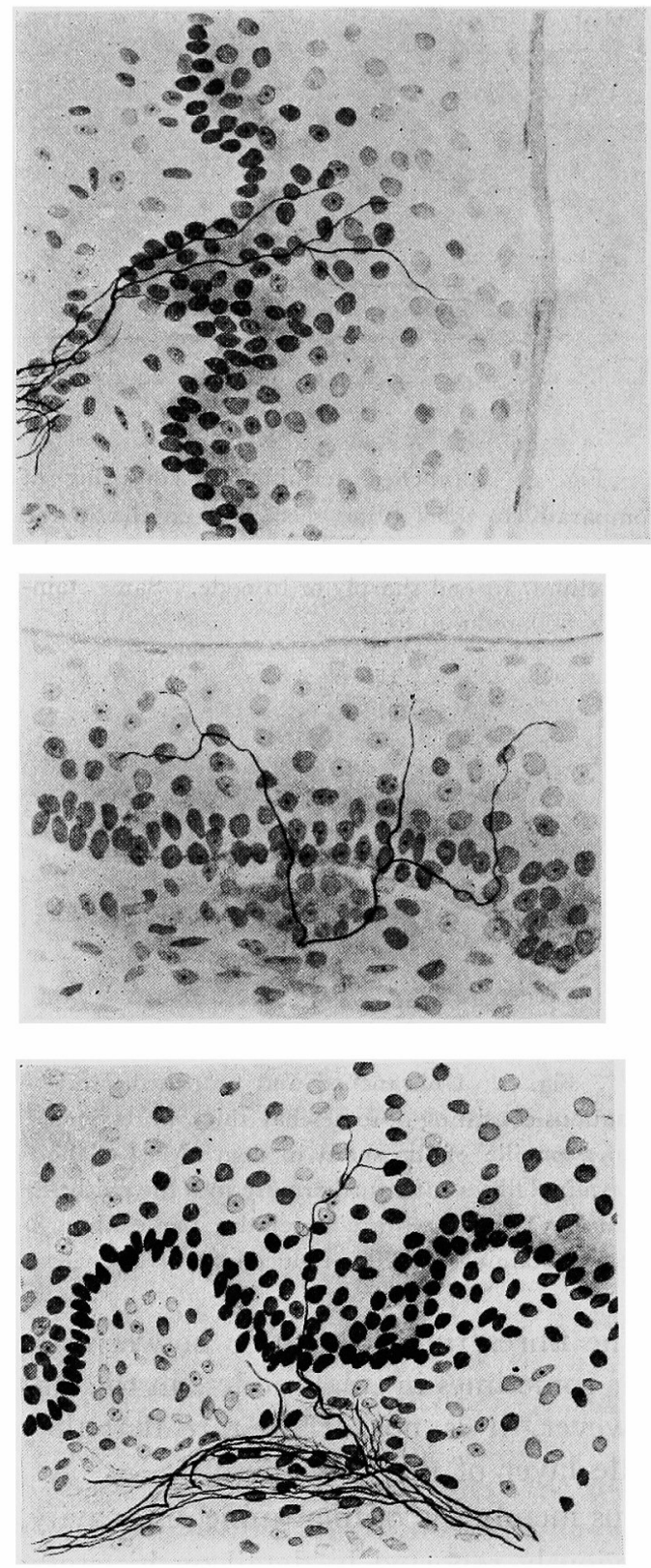

Fig. 7. Branched intraepithelial sensory termination consisting of somewhat thick fibres seen in the snout in dog. All of the terminal fibres end sharply. Same staining, $X$ 600, reduced to $\frac{1}{2}$.
Fig. 8. 2 bifurcated intraepithelial fibres seen in the snout in dog. Same staining, $\mathrm{X} 600$, reduced to $2 / 3$.
Fig. 9. Branched intraepithelial fibre seen in the snout in dog. The terminal fibres end sharply. Same staining, $\mathrm{X} 600$, reduced to $\frac{1}{2}$.

the lips, the gums and the cheeks.

Nerve supply of lips and cheeks in dog:

Upon this subject, we have had no investigations with mammals as objectives, but there have been the brilliant studies by Fujii ${ }^{17)}$ and Suga ${ }^{18)}$ of this 
laboratory respectively conducted with human adults and embryos. The human lips, as reported by Luschca, ${ }^{19)}$ Neustätter ${ }^{20)}$ and others, consist of the pars cutanea, the pars transitionis and the pars mucosa. The transitional part represents the part lying between the outer skin part and the inner mucosa part and are divided into the inner and the outer zones by a conspicuous furrow in the latter stage of embryonic life. The inner zone, due to the formation of lip downs on its surface, has been called the pars villosa and the outer zone, the pars glabra, as it remains smooth (Luschca).

As in the lips also, the development of sensory fibres goes with the development of the papillae, the histology of this part has been studied by Fujiiit) and Suga ${ }^{18)}$ very minutely. Such studies have revealed that the epithelium suddenly increases in height as it goes over from the pars cutanea to the outer zone of the transitional part, directly beneath which regularly aligned small papillae are formed, which are not observable in pars cutanea. Upon reaching the inner zone, the epithelium is rapidly thickened again, and the papillae become also more powerful. However, in pars mucosa, both the height of the epithelium and the development of the papillae become considerably poorer. The epithelium is composed of stratified flat epithelium, with corneous plate in the outer, and non-corneous in the inner, zones. This histological picture is especially vivid in the latter stage of human embryonic life, but that in adult lips also shows a glimpse of such a genesis, that is, there is no essential histological difference between the embryonic and adult lips.

The development of the sensory fibres in human lips is most emphasized in the inner zone of the transitional part, next in the outer zone thereof, the mucosa part standing in an inferior position to the two foregoing in this respect. Thus, the sensory terminations are best developed in the inner zone where the papillae are also most strongly developed, succeeded by the outer zone, where the papillae are in good formation, in spite of the low epithelium, but is poor in the mucosa part, where the epithelium is rather thick but the papillae are not so well developed.

The sensory terminations in the lips are chiefly of branched type in the outer zone, but also some non-capsulated glomerular endings, or endings resembling the genital nerve bodies are found existing here. Intraepithelial fibres originating in these endings are also found in many places. In the inner zone, the sensory fibres are better developed, their endings consisting of more complex branched and glomerular endings, accompanying a small quantity of intraepithelial fibres. In the mucosa part, however, there are found some simpler glomerular and quasi-genital bodies, but no intraepithelial fibres. In human embryo, no corpusclar termination is yet formed in the lips.

The histological picture of the lips of my baby dogs resembles strontly that in human embryo. The pars cutanea consists of a corium with numerous hair follicles and a thin corneous epidermis without papillae. Interestingly enough, the canine lips are also divided into the inner and outer zones at the transitional part, by a shallow furrow, as is the case with human lips. The outer zone occupies only a small space, and its epithelium is about 3 times that 
in the pars cutanea in thickness, is composed of a stratified flat epithelium with corneous plate, and considerably well-developed papillae are formed against it. Upon going over from the outer to the inner zone, the epithelium grows promptly in thickness, reaching 4 or 5 times of the thickness in the outer zone at the middle part of the inner zone. The epithelium consists of non-corneous stratified flat epithelium without corneous plate, and villi-like epithelial cell scales, as seen in human embryo, are found on its surface. The formation of papillae becomes stronger, especially increasing in height. This part occupies a far larger space than the outer zone, and the height of the epithelium grows gradually thinner as it goes over into the mucosa part. In the latter part, the epithelium decreases in height, so that it becomes as low as in the outer zone. In contradistinction to the human counterpart, there is little formation of papillae. In the cheek mucosa lying exterior to the lips, the epithelium is high and provided with well-developed papillae in the part above the level corresponding to the rima oris, as in human cheek, but in other parts, no papillae are formed and the epithelium is also much lower.

As the histology of the mucous membrane of the lips and cheeks in dog in its similarity to that in man clearly reveals, the sensory supply of these parts is also much the same in man and dog. So, the inner zone of the transitional part, the outer zone thereof and the mucosa part of the lips are best supplied, in the order named. However, the number and the terminal formation of the sensory fibres are much inferior in dog lips to those in man. Similar inferiority has been demonstrated in dog tongue by Okano, ${ }^{11}$ too. In the cheek mucosa, the parts above the level corresponding to the rima oris provided with papillae are rich in sensory fibres, but the other parts are very poorly supplied.

The sensory fibres in the transitional part of the canine lips, both in the outer and inner zones, end in papillae, generally in comparatively simple branched endings, but sometimes also in simplest unbranched endings, no such complex branched and corpusclar endings as found in man being observable. Intraepithelial fibres are found in the outer zone, consisting mostly in thin smooth-surfaced fibres, often sending out rami (Fig. 10), and quite as often running out unbranched, to end sharply or in small nodes in the upper layer of the epithelium.

Innervation of hard palate in dog: There have been many studies on the nerve supply of the hard palates of mammals as cited in the report on that in cat and hedgehog by us, ${ }^{21)}$ but these studies are of rather dubious value owing to the imperfection of staining methods. So, we have made a research on the same question of the two kinds of mammals mentioned above, utilizing the Seto's impregnation, and similar to those in man in distribution, but as much as the palates of these animals show histological pictures simpler than in man, the number and terminal formation of 
sensory fibres were also poorer. The majority of these sensory terminations in the papillae were unbranched, as well as the intraepithelial fibres.

The sensory fibres in the hard palates of my baby dogs also were poorer than in man, both in number and in terminal formation, as was the case in cat and hedgehog, but somewhat better than in cat in both respects. In cat, the large majority of the sensory terminations were merely simple unbranched endings, but in dog, the terminations often consisted in more complex branched endings, as shown in Fig. 11. Intraepithelial fibres

Fig. 10

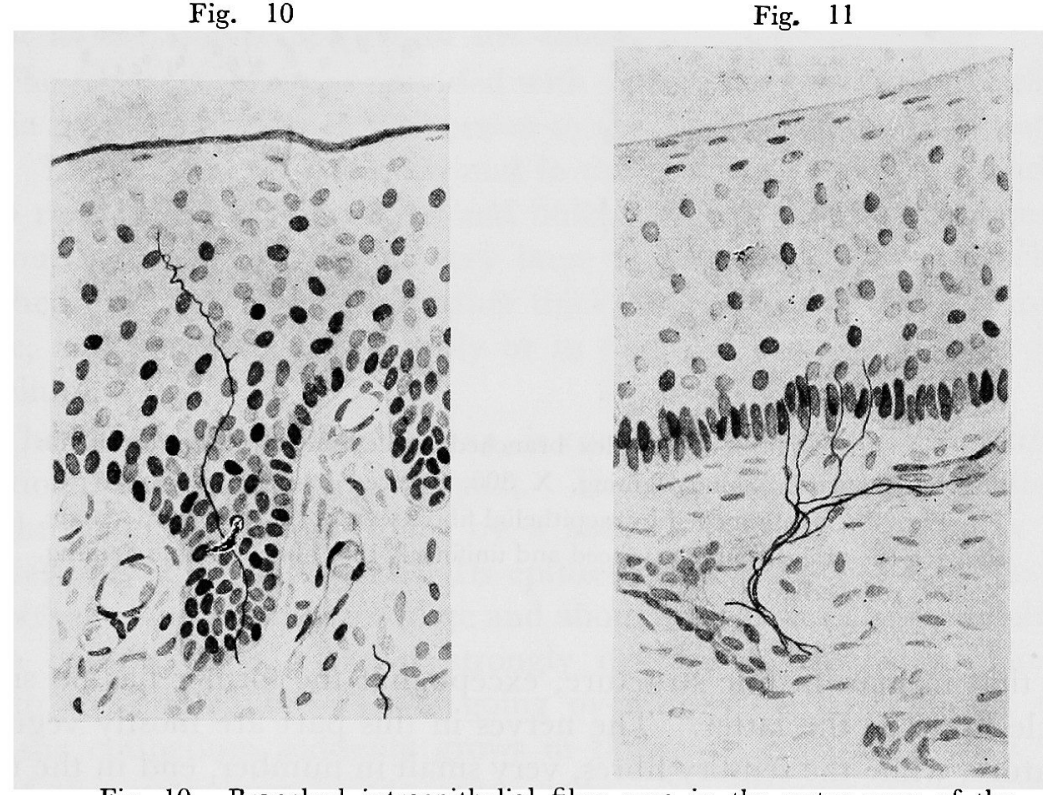

Fig. 10. Branched intraepithelial fibre seen in the outer zone of the transitional part of the dog lip. Its running course through and between the epithelial cells is seen clearly. Same staining, X 500, reduced to $2 / 3$.

Fig. 11. Somewhat complex branched termination formed beneath the epithelium of the dog hard palate. Most of the terminal branches enter the basal layer of the epithelium, to end sharply. Same staining, X 400, reduced to $2 / 3$.

were more numerous in $\operatorname{dog}$ and they were not always represented by simple unbranched terminations (Fig. 12) as in cat but often by branched endings (Fig. 13). These also consisted of uniformly thin smooth-surfaced fibres running through or between the epithelial cells and ending sharply or in small nodes in the upper layer of the epithelium, as was the case in cat and hedgehog.

\section{Summary}

The mucous membrane of the canine nasal cavity is nearly the same 
Fig. 12

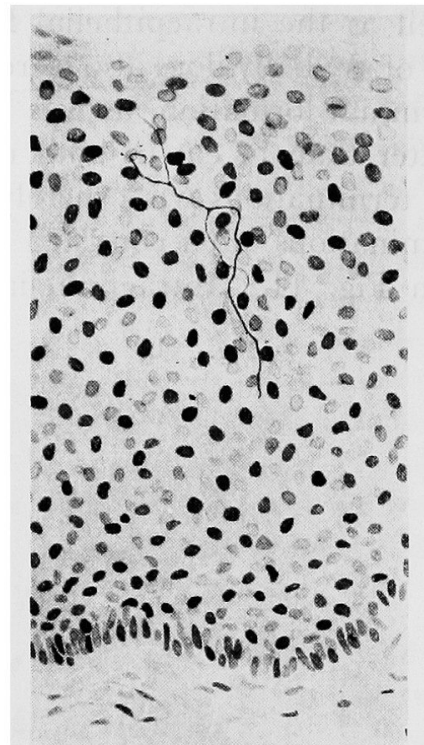

Fig. 13

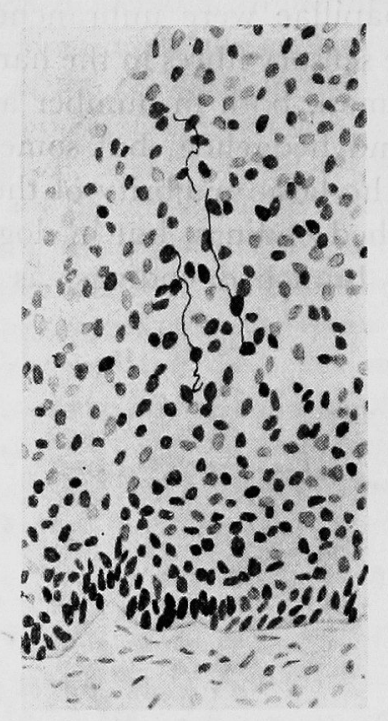

Fig. 12. Somewhat complex branched intraepithelial fibre seen in the $\operatorname{dog}$ hard palate. Same staining, X 300 , reduced to $\frac{1}{2}$.

Fig. 13. Unbranched intraepithelial fibres seen in the dog hard palate, showing the typical smoothsurfaced and uniformly thin fibres. Same staining, $\mathrm{X} 300$, reduced to $\frac{1}{2}$.

with that in man in fine structure, except that the former has no smooth muscle fibres as the latter. The nerves in this part are mostly vegetative in nature, while the sensory fibres, very small in number, end in the tunica propria in simple endings, without further entering into the epithelium.

The minute structure of the canine vestibulum nasi is also similar to that in man, except that the former has no pars cutanea with vibrissae, the vestibulum in the front partly going over to the snout, partly to the haired outer skin. The mucosa of the vesstibulum is divisible into the corneous part with corneous plate and the non-corneous part, as in the human vestibular mucosa part, but the development of nasal glands is much inferior in the former.

In the submucosa of the vestibulum nasi in dog there are seen many sensory fibres, most of which run toward the corneous mucosa part, to end chiefly with branched endings in the papillae. More intraepithelial fibres are found than in the human counterpart, being in best development at the entrance of the vestibulum. These fibres originate partly in the branched endings in the papillae and partly in single fibres directly penetrating the epithelium. They either end unbranched or branched, 
are mostly smooth-surfaced and thin, but more rarely somewhat thicker, and run through or between the epithelial cells, to end sharply or sometimes in nodes in the upper layer of the epithelium.

The snout in dog presumably represents the foremost part of the vestibulum. The epithelium is similar to that of the corneous mucosa part of the vestibulum, being composed of stratified flat epithelium with corneous plate and somewhat greater in height than the latter. The development of papillae is much stronger than in the vestibular corneous part and their arrangement is very regular. No nasal gland is found formed in the tunica propria of the snout.

The snout is far better provided with sensory fibres than the vestibular mucosa part, apparently in proportion to the good development of papillae here. The sensory fibres mostly end in the papillae as branched endings, but in rarer cases, as capsulated end bulbs. Often enough, these endings send out intraepthelial fibres, very large in number, most of which are branched. Some of them are rather thick, though mostly they are minute in size, and generally end sharply or in nodes in the upper layer of the epithelium.

The canine lip, as that in man, is composed of the cutanea part, the transitional part and the mucosa part. The transitional part is subdivided into the outer and inner zones by a shallow furrow. The outer zone occupies only a narrow space. Its epithelium is composed of a stratified flat epithelium with corneous plate and about 3 times thick as the epidermis of the cutanea part. Rather strongly developed papillae are formed beneath the epithelium. Upon going over from the outer to the inner zone, the epithelium suddenly grows in thickness, reaching 4 to 5 times that of the epithelium in the outer zone. No corneous plate is found herein, and villi are formed on the surface. The papillae here are tall and well-developed. The inner zone occupies a far larger area than the outer. Its epithelium diminishes in thickness as the mucosa part is approached, and its height becomes nearly equal to that of the outer zone upon going over to the mucosa part. In the mucosa part, no papillae are formed, unlike that in human lips. Of the mucous membrane of the cheek outside the lip mucosa, only the part corresponding to the level above the rima oris contains papillae.

The sensory supply of the mucous membrane of the lips and cheeks in dog is similar to that in man, the inner zone of the transitional part, the outer zone thereof and the mucosa part being best innervated, in the order named. But the number and the terminal formation of the sensory fibres are much inferior to man, corresponding to the simpler histological picture of the canine mucous membrane. Of the mucosa of the cheek, 
only the part corresponding to the level above the rima oris, which is provided with papillae, is rich in sensory fibres.

The sensory terminations in the transitional part of the lip are chiefly represented by simple branched endings, and no such complex endings and terminal bodies as found in man are formed here. Intraepithelial fibres are seen only in the outer zone, as smooth-surfaced fine fibres ending branched or unbranched, with their terminal rami ending sharply or in small nodes.

The sensory fibres in the canine hard palate are much poorer in development than in man, as is the case with cats and hedgehogs. They are, however, greater in number than in cat and their terminations show more complexity. Rather complex branched terminations are found in the papillae, and the number of the intraepithelial fibres is also larger than in the feline couterpart.

\section{References}

1) Seto \& Sada, Tohoku Igaku Zassi (Jap.), 1944, 34, 199.

2) Sato, ibid., 1949, 40, 8.

3) Abrahám, Arch. Zool. Italiano, 1931, 16, 717.

4) Suga, 1. \& Suga, Y., Tohoku Igaku Zassi (Jap.), 1950, 45, 337.

5) Szymonowicz, Arch. mikr. Anat., 1895, 45, 624.

6) Trejakoff, Z. wiss. Zool., 1911, 97, 314.

7) Merkel, Cited by Hashizume (12).

8) Botezat, Arch. mikr. Anat., 1903, 761, 730.

9) Kadanoff, Z. Anat. u. Entw. g., 1924, 73, 431.

10) Boeke, Z. mikr. -anat. Forsch., 1933, 33, 47.

11) Akkeringa, ibid., 1930, 19, 183.

12) Hashizume, Kaibogaku Zassi (Jap.), 1938, 12, 715.

13) Huss, Z. wiss. Zool., 1898, 63, 1.

14) Cybulsky, ibid., 1883, 39, 653.

15) Mornono \& Tsuda, Tohoku Igaku Zassi (Jap.), 1950, 46, 569.

16) Okano, Tohoku J. Exp. Med., 1952, 56, 169.

17) Fujii, Tohoku Igaku Zassi (Jap.), 1948, 38, 123.

18) Suga, ibid., 1950, 45, 437.

19) Luschca, Möllendorff's Handbuch, V, Berlin J. Springer, 1927.

20) Neustätter, Jena. Z. Naturwiss., 1892, 29, 345.

21) Ogasawara, Abe \& Sato, Tohoku J. Exp. Med. 1954, 59, 371. 